
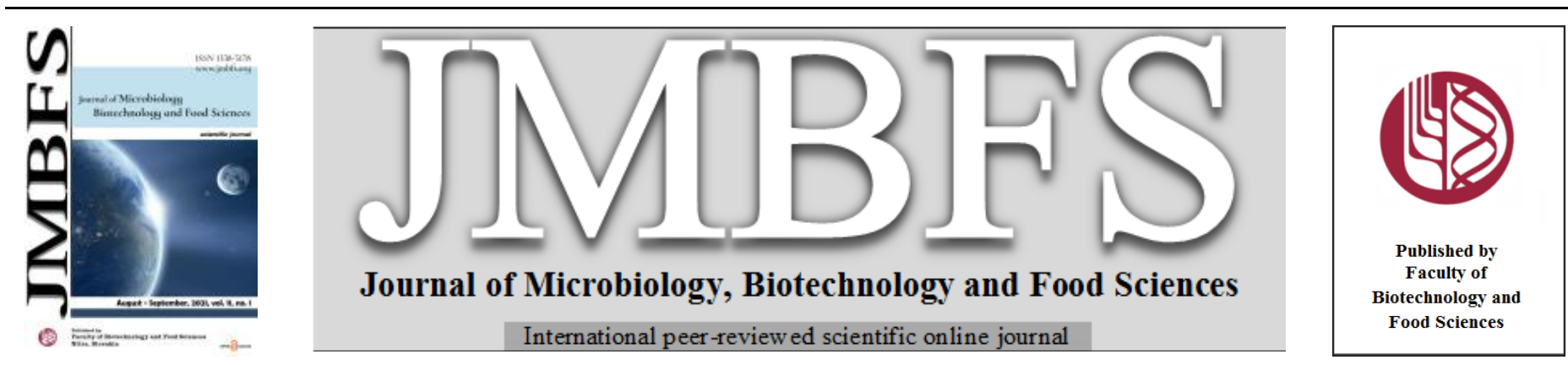

\title{
COVID-19: PERVASIVENESS OF COMORBIDITIES WITH EMPHASIS ON CANCER, PATHOGENESIS AND PALLIATIVE CARE MEASURES
}

\author{
Sonali Dhaundiyal ${ }^{1}$, Jigisha Anand ${ }^{2}$, Nishant Rai $*^{3}$ \\ Address(es): Nishant Rai, \\ ${ }^{1}$ Graphic Era Deemed to be University, Research Scholar, Department of Biotechnology, Bell Road 566/6, 248002-Dehradun, Uttarakhand, India. \\ ${ }^{2}$ Graphic Era Deemed to be University, Assistant Professor, Department of Biotechnology, Bell Road 566/6, 248002-Dehradun, Uttarakhand, India. \\ ${ }^{3}$ Graphic Era Deemed to be University, Professor, Department of Biotechnology, Bell Road 566/6, 248002-Dehradun, Uttarakhand, India.
}

*Corresponding author: nishantrai1@gmail.com

https://doi.org/10.15414/jmbfs.4069

ARTICLE INFO

Received 12. 12. 2020

Revised 3. 3. 2021

Accepted 3. 3. 2021

Published 1. 8. 2021

Short communication

open $\mathcal{O}$ access

\begin{abstract}
In retrospection and review of the available literature, cancer patients are regarded as highly susceptible to the incidence of the ongoing 'COVID-19' pandemic. Other comorbidities including hypertension, diabetes, kidney problems, high cholesterol are found to be having severe effects on COVID-19 related mortality. Reports associating these diseases with COVID-19 must be carefully elucidated not just because these comorbidities induce severity in COVID-19 patients but also as several other factors increase the risk of occurrence of this pandemic. From vigorous screening to advance researches regarding this association as well as the still elusive mechanism of its pathogenesis, from clinical management to implementation of national and international guidelines, from adopting a dynamic approach to tackle the co-infection to having resourceful hospitals and medical care facilities, a structured and methodical proposition involved in the development of therapeutic strategies promise a better approach to target COVID-19.
\end{abstract}

Keywords: Acute Respiratory Distress Syndrome, Cancer, COVID-19, co-morbidity, pandemic, SARS-CoV-2

\section{INTRODUCTION}

We read an interesting article in TheActuary Magazine entitled 'The Comorbidity Question', which reinstates the argument, speculated by many- Would majority of COVID-19 victims have died this year anyway? While there may be opposing views to this statement, it necessitates the management of palliative care patients and implementation of proper treatment and isolation measures to mitigate the risk and decimation COVID-19 poses.

The authors calculated life expectancies for people according to age, gender, disease history, lifestyle (body mass index, smoking habits) and various other factors and concluded that the aforementioned claim is unsubstantiated and very dangerous from a public health perspective. The authors stated their views that people who would be alive now in the absence of coronavirus (in great majority of cases) would still be alive in several years' time (TheActuary 2020).

As of $31^{\text {st }}$ October, 2020, the total number of coronavirus cases across the world has crossed the 45 million mark and has claimed the lives of more than 11 lac people so far. This view that people with comorbidities are more susceptible to COVID-19 is supported and evidenced by many researchers. The clinical signs of COVID-19 infection range from mild to severe ones with fatigue, cough, fever, dyspnoea included in the former and the severe cases can lead to fatal complications like Acute Respiratory Distress Syndrome, septic shock, thromboembolism and multiple organ failure (end organ diseases) (Machhi et al., 2020). Sometimes asymptomatic phase is also confirmed and found to be contagious in individuals called super-spreaders (Dariya et al., 2020).

An intensive screening and reviewed study conducted by two authors and published in American journal of infected control deduced $57.7 \%$ of the patient population with one or more chronic comorbid condition in a cohort of 22,753 COVID-19 positive patients under 27 reviewed studies from various nations (Thapa et al., 2020).

Comorbidities frequenting the population included Cardiovascular disease, Hypertension, Diabetes, Chronic obstructive pulmonary disease, Cancer, Chronic kidney disease, and others. WHO statistical data shows that $71 \%$ of the global million deaths in 2016 were due such non-communicable diseases making them the leading causes of mortality worldwide (WHO https://www.who.int/data/gho/data/themes/topics/topic-details/GHO/ncdmortality). Given the limited amount of evidence and case studies on a lesser number of patients, an insightful approach and well-structured studies are needed to investigate the apparent relatedness between comorbidities and COVID-19.
This article asserts and identifies studies from various sources defining vulnerable patient populations with comorbidities and their relation with the ever threatening COVID-19 complications. The risk for such patients is high and owing to the high risk, people with one or more comorbid diseases must follow effective isolation protocols especially in areas with high COVID-19 infection rate. As we eagerly await the genesis of some antiviral drug or vaccine to combat this disease on the forefront, the patients who are on a vulnerable edge must be met with concerted intervention strategies of risk reduction and considerate treatment regime in order to battle this plight.

Literature reviews have been published to date which probed into the prevailing and underlying chronic illnesses and their significant associations with COVID19 and ranked them according to their fatality rates. The individuals with comorbidities in comparison to those without any may be subjected to 10 times higher risks of severe outcomes- as evidenced by composite data in the current scientific literature (Thapa et al., 2020).

\section{ORGAN FAILURE AS A RESULT OF COVID-19}

As discussed earlier, the typical clinical signs of COVID-19 include fever, fatigue, cough, and some patients have also developed dyspnoea. Atypical symptoms like nausea, vomiting, diarrhoea, sputum with blood have also been detected (Guo G et al., 2020). Olfactory and gustatory impairment is strongly associated with COVID-19 infection and is recovered within weeks in most patients (Yan et al., 2020).

Beta coronavirus is an enveloped, single-stranded RNA isolated from different animal species and resulting in enteric and respiratory illness in the host. The club-shaped, corona like spike projection is made up of S-protein which binds host cell receptors thereby facilitating viral infection. SARS-CoV and 2019nCov/ SARS-CoV-2 were found to share a better sequence homology than with MERS-CoV (Xu et al., 2020).

ACE2 receptors are present on the target cells, which facilitate the entry of the virus through its interaction with $\mathrm{S}$-protein and its subsequent pathogenesis ACE2 is found expressed on the cells of Type I and Type II alveolar epithelium, upper respiratory tract, myocardium, endothelium, renal tubular epithelium, testis, gastrointestinal system with Type II alveolar cells (AT2) showing considerably high expression of ACE2 receptors, rendering them the primary receptors for SARS-CoV-2 (Dariya et al., 2020). 
A novel observation has been made that ACE2 shows comparably high levels of expression in the gastrointestinal tissues, like ileum, duodenum, jejunum, colon thereby suggesting further experimentation and inquiry for evaluation of the role of ACE2 in gastrointestinal pathophysiology (Hammer et al., 2002).

Moreover, the physiologic role of ACE2 in cardiac function has been elucidated in ACE2 knockout studies which revealed that its expression increases after myocardial infarctions which could contribute to our overall understanding of COVID-19 effects and therapeutics development relevant to heart failure (Burrell et al., 2005).

An observational study, comprising of 52 critically ill adult patients (with a mean age of 59.7 years) with SARS-CoV-2 pneumonia was carried out for 1 month in Wuhan Jin Yin-tan hospital (Wuhan, China). Upon admission to ICU, 32 death were reported in 28 days, among the survivors- 35 patients had organ function damage with Acute Respiratory Distress Syndrome (ARDS), 15 were reported with acute kidney injury, 12 with cardiac injury, while liver dysfunction was observed in 15 and pneumothorax condition in one (Yang et al., 2020).

In a study to determine the expression and distribution of ACE2 in the adult human heart, single nuclear transcriptome analysis revealed that pericytes (required for myocardial microcirculation) displayed high and specific expression of ACE2 suggesting that they might be a potential SARS-CoV-2 virus targeted host cell in the human heart. Damage to the virus-infected cells leads to the disruption or injury of the myocardial pericytes hence causing microvascular dysfunction. Transcriptome and proteome analysis data revealed that basic cardiovascular disease patients are most susceptible to SARS-CoV-2 infection and may even progress to critically ill condition and death (Chen $\boldsymbol{e t}$ al., 2020).

Moreover, various amino acid residues of the RBD (Receptor Binding Domain) of the mature spike protein of SARS-CoV-2 that may be critical for binding with ACE2 receptor have been discovered. Thus, while designing and docking potential drugs against novel coronavirus, these amino acid residues and their affinity towards ACE2 receptor are to be considered (Chen $\boldsymbol{e t}$ al., 2020).

Diffuse alveolar damage and acute respiratory failure are most common in severe cases of COVID-19 patients.

To understand the effect of SARS-CoV-2 on kidney function, Cheng et al. performed a cohort study of 701 COVID-19 patients admitted to a tertiary teaching hospital in Wuhan. The prevalence of Acute Kidney Injury (AKI) was reported to be in $6.7 \%$ of the patients with SARS, $91.7 \%$ of which eventually died. Among other kidney abnormalities, serum creatinine and blood urea nitrogen were found to be elevated; incidences of proteinuria and haematuria were also seen (Cheng $\boldsymbol{e t}$ al., 2020). AKI was associated with high mortality and morbidity- about 5.3 times higher as compared to the patients without AKI. The kidney- lung crosstalk theory states that the experimental evidence of ACE2 expression in renal epithelial cells alongside expression in respiratory organs in humans could be the major cause of kidney disease in COVID patients. Additionally, virus-induced cytokine storm started in the lungs could be accelerated and progressed to the kidneys and impair kidney function, eventually leading to multi-organ failure or even death (Li et al., 2020).

\section{OTHER COMORBIDITIES}

Individuals with diabetes have a high risk of respiratory infections and even present severe disease cases when infected with respiratory viruses. A multicentred study of 150 patients with 68 death cases and 82 discharged cases in Wuhan showed that co-morbidities were identified as a significant clinical predictor of severe patient outcomes and COVID-19 related mortality (Ruan $\boldsymbol{e}$ al., 2020).

The Chinese Centre for Disease Control and Prevention recently published the largest case series (a report of 72,314 cases of Coronavirus disease) in mainland China whereby the case fatality rate (overall-2.3\%) was found substantially increased in the patients with pre-existing comorbid conditions. Given that the overall CFR in this study was $2.3 \%$, it was elevated to $7.3 \%$ in diabetic patients making this assumption logical that coexisting comorbidities like heart disease, kidney disease, diabetes and advanced age- all contribute to the increasing severity of the COVID-19 disease (Wu et al., 2020).

The study of hypertension as an emerging risk factor for COVID-19 associated pneumonia and reduction in pulmonary function was carried out via UK Bio bank data analysis. (Out of 107,310 participants with hypertension enrolled, 3\% developed pneumonia afterwards). This study provides evidence that elevated blood pressure directly influences the risk for pneumonia. Vigilant blood pressure monitoring and control, alongside other measures could help reduce this risk (Zaki et al., 2020).

According to systematic reviews and meta-analysis data, the risk of respiratory infection and mortality is significantly higher in patients with hypertension. Studies that focus on hypertension as a solid indicator of COVID-19 severity, continuation of medicinal treatments of this comorbidity to lower severity risk have been conducted and most health organizations have generally recommended hypertension treatment to be continued in the case of COVID-19 infection (Zaki et al., 2020). In USA, according to data obtained from a hospital report, underlying health conditions were found in 71\% (732/1037) of COVID-19 patients and 94\% (173/184) of reported deaths (Clark et al., 2020).

\section{MECHANISM OF ACE2 ACTION}

Several experimental studies previously carried out have implicated that the renin-angiotensin system (RAS) has been instrumental in the pathogenesis of Acute Respiratory Distress Syndrome (Wösten-Van Asperen et al., 2013). The classical RAS-ACE-Ang II-AT1 pathway has a counter regulatory pathway modulated by angiotensin-converting enzyme -2 by converting Ang (Angiotensin)-II into Ang-(1-7). Ang-(1-7) activates Mas receptor and exerts an anti-proliferative, anti-inflammatory effect and works opposite to the classical RAS pathway by promoting vasodilation (Wu et al., 2020) (Figure 1).

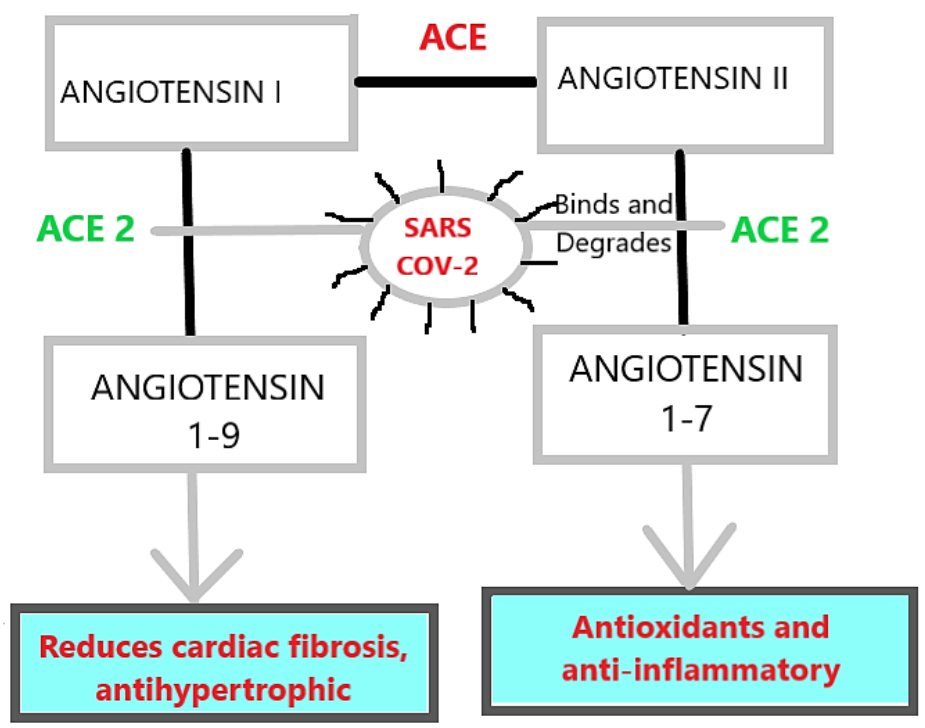

Figure 1 Pathogenesis of the SARS-CoV-2 virus, its potential receptor targets and subsequent effects

ACE (angiotensin-converting enzyme), a dicarboxypeptidase cleaves Ang-I to generate Ang-II after which Ang-II binds to Angiotensin II receptor type 1 receptor (AT1) and plays its role in triggering inflammation, vasoconstriction and fibrosis. Alternatively, ACE2, a monocarboxyl peptidase, inactivates Ang-II and generates Ang-(1-7) which binds to the MAS receptor and induces a negative regulatory effect to the classical pathway.

An animal model study indicating the involvement of the 2 enzymes i.e. ACE and ACE2 in ARDS demonstrated the pulmonary imbalance of ACE/ACE2 activity- with markedly increasing levels of ACE and decreasing expression of ACE2 in the ARDS model. Results showed that ARDS develops due to repletion in the levels of Ang II and reduction in Ang (1-7) (Wösten-van Asperen, Lutter et al., 2011).

In recent in vitro and molecular model studies, it has been experimentally proven that ACE2 is likely the cell receptor for 2019-nCoV. In an experiment confirming pathogenicity of 2019-nCoV, through its infection on hACE2 transgenic micehistopathological studies revealed accumulation of macrophages, lymphocytes in alveolar cavities, presence of viral antigens in these macrophages, alveolar and bronchial epithelia. No such observation was found in wild type mice infected with SARS-CoV-2 (Zhao et al., 2020).

The recent speculation that ACE2 is down-regulated by binding of the virus with ACE2 receptor has brought forth an array of strategies proposed by the researchers to treat this infection by targeting ACE2 expression in its host cells. These include- inhibition of trans membrane-protease serine 2 (TMPRSS2) activity which carries out initial spike protein priming to facilitate viral entry, inhibiting the interaction between ACE 2 and $\mathrm{nCoV}$ by blocking its receptor with antibodies or small molecules and by using soluble ACE2 which not only binds competitively with the virus and neutralizes it but also restores cellular ACE2 activity of negatively regulating RAS to protect the lung from injury (Zhang - et al., 2020). Therefore, ACE2 is proposed to play a dual role in SARS induced lung injury- in which initially, it acts as a receptor for the virus, then its decreased availability promotes lung injury (Zhang et al., 2020).

\section{ARE INCIDENCES OF COVID-19 IN CANCER PATIENTS HIGH?}

It is hypothesised that due to an impaired immune system, cancer patients are facing a higher risk of infection from this viral pandemic.

In the midst of this global contagion which has taken people to their heels, cancer patients are included amongst the 'most vulnerable groups', especially in the highly endemic COVID-19 areas. Limited data, unstructured surveys, multifariousness of this infection and uncertain treatment regimens are few but some critical issues demanding urgent attention. Several questions leave us 
wondering and in doubt as to whether COVID-19 cancer patients should undergo different clinical therapy or continue with the conventional antitumor treatment and what is the possible worst-case scenario in such patients: severe respiratory distress or death from pneumonia? Our goal is to redress the address made in certain preliminary surveys and case studies as well as reviewed literature published in many journals of high esteem that--- it is the need of the hour to probe into the critical condition of the COVID-19 infected cancer patients.

In a review of the medical records and other data of 1524 patients with cancer admitted to the Zhongnan Hospital of Wuhan University, Wuhan being the epicentre of this deadly pandemic, $0.79 \%$ of COVID-19 infection rate was observed. The association between SARS-CoV-2 infection and age factor and non-small cell lung cancer (NSCLC) were also determined. Out of the 1524 cancer patients, 8 patients screened for the virus were $>60$ years old and 228 patients had NSCLC (Yu et al., 2020).

Nonetheless, a small sample size of patients did not indicate that age was associated with susceptibility to infection. Studies on a larger cohort with cancer might resolve these potential associations.

Another retrospective study carried out in three hospitals in Wuhan namely, TongjiSino-French New Town Hospital, Union Red Cross Hospital, and Union West Hospital wherein hospitalized cancer patients infected with COVID-19 were identified in a one month study and new COVID-19 patients previously diagnosed with cancer were enrolled

Among 28 cancer patients, lung cancer was predominant in 7 , followed by oesophageal cancer in 4 and breast cancer in 3 . Ten patients were diagnosed with stage IV cancer. In addition to cancer, 11 patients had at least one or more coexisting chronic diseases. Abnormal radiological findings like Ground-glass opacity, Patchy consolidation and reticular appearances were found in most patients. CT images revealed decreased lung volume along with features of pneumonia in lung cancer patients (Dariya et al., 2020).

Treatment options for patients included oxygen therapy, invasive mechanica ventilation due to hypoxia and endotracheal intubation with the percentage of patients in descending order. Among the antiviral agents prescribed were arborol, lopionavir/ritonavir, ganciclovir, ribavirin, empirical antibiotics, intravenous immunoglobin and corticosteroids.

Early diagnosis of this disease in cancer patients can be challenging due to the similarity in symptoms between the infection and underlying cancer, especially in lung cancer patients. Presence of radiographic features in cancer patients similar to those of SARS-COV-2 infection which can be misleading, atypical radiological features of lung adenocarcinoma alongside few other reasons.

Cancer being a heterogeneous disease, this therapeutic dilemma must be resolved considering multiple factors including the type of therapy used, biological features of the tumour, the extent of comorbidities, concomitant medications an treatment characteristics (resistance), patient clinical condition and age, the effect of delay or modification of cancer therapy on its recurrence, among others (Larson et al., 2020).

\section{CANCER CARE DURING THE PANDEMIC}

As it is a fact that old age and co-existing co-morbidities are potential risk factors for severe and critically ill COVID-19 patients and the patients with cancer are highly susceptible to viral infections as compared to healthy ones owing to an immune-compromised status alongside the malignancy. Hence, this unprecedentedly evolving pandemic poses special challenges for medical oncologists and their patients including the diagnostic challenge in cancer patients, clinical and abnormal radiological findings especially in patients undergoing chemotherapy, tumour characteristics and progression requiring medical prowess of the oncologists. Moreover, optimal management of the frail patient population with cancer concurrently affected by COVID-19 must be strived for by professionals and emphasis on palliative patient care and cancer research which has been hindered during COVID-19 crisis should be highlighted (Lambertini et al., 2020).

A group of young oncologists of Italy, where after China, the COVID-19 mortality rate shot up tragically, suggested some measures to adapt to the crisis faced by the nation with more than 1,00,000 cases by the end of March, 2020 . These measures included a delay of some anticancer treatment based on a caseby-case evaluation of the risk/benefit ratio, prioritizing urgent and aggressive tumour situations, withholding treatment of clinically stable patients, continuance of adjuvant and neo-adjuvant chemotherapy with the main treatment, encouraging follow-up of patients (Xu et al., 2017).

Decreased ACE2 expression and signalling during SARS-CoV-2 infection leads to acute lung injury (ALI)/ acute respiratory distress syndrome (ARDS) followed by cytokine-induced oxidative stress and inflammation. The use of angiotensin converting enzyme (ACE) inhibitors and angiotensin II type-I receptor blockers (ARBs) is a subject of debate by many, however, its ability to increase ACE2 expression and activity, anti-angiogenic and tumour-regressive effects support the argument favouring its use (Larson et al., 2020).

Due to their potential anti-tumour effects by inhibiting angiogenesis and tumour progression, the continuation of ACEIs/ARBs is recommended especially in cancer patients (Soy et al., 2020). As SARS-CoV-2 binds to ACE2 for target cell entry, so ACE 2 overexpression might suggest intensified viral infection.
Conversely, research studying ACE2 mechanism indicates that ACE2 overexpression might counteract inflammation and vasoconstriction thereby reducing the incidence or mortality rates of COVID-19 associated ALI/ARDS (Larson et al., 2020).

In a prospective observational study, aimed at the description of clinical and demographic features of COVID-19 patients with cancer, eligible patients were enrolled in UK Coronavirus Cancer Monitoring Project (UKCCMP). Out of 800 patients that have been examined, 52\% of the patients had a mild COVID-19 while $28 \%$ of the patients died. Age, gender, and comorbidities were the main factors driving COVID-19 related mortality. No substantial evidence of the effect of chemotherapy and anti-cancer treatment on mortality from COVID-19 disease was observed in this study (Lee et al., 2020).

It has been established that in severe cases, immunologic complications like macrophage activation syndrome leading to hyper-inflammatory immune response also called cytokine storm is developed (Soy et al., 2020). A hypothesis proposed by Akula et al., states that COVID-19 survivors might be at risk of developing cancer as the SARS-CoV-2 induced cytokine storm might activate signalling cascades leading to abnormal cell growth and impairment of body's immune system against tumours. Future in vitro and in vivo studies investigating the association laid by this hypothesis is required (Akula et al., 2020).

An effective vaccine or treatment might take time to be commercially available, although drugs like hydroxychloroquine, chloroquine, arbidol, remdesivir, favipiravir and several anti-tumour agents (thalidomide) are being investigated and recommended as a promising medication for COVID-19 and underlying cancer (Larson et al., 2020).

\section{CONCLUSION}

The crisis of COVID-19 with surging cases and a devastating death toll alarmed people of an impending socio-economic and existential crisis. Tight restrictions imposed on movements have brought economic progress to a standstill and has resulted in the loss of many jobs

Various social, economic and health sectors are concerned with the daily, intensifying death peaks from the major epicentres all around the globe and a plethora of reports and superfluous literature examining the risks associated with COVID-19 diagnosis and mortality are available. The higher age group has been established as a well-observed risk factor in severe cases, though, precautionary measures by and for the younger generations has been emphasised by many. Guidelines specifying risks associated with severe COVID-19 cases have been issued by WHO along with public health agencies in countries like UK and USA. Therefore, a strategic perspective should be adopted keeping in view the current COVID-19 crisis in relation to the immune-compromised status of patients with comorbid diseases. Extensive and optimal treatment should be provided to those caught up with the age factor or one or more comorbidities. Researches must be conducted to establish a relationship between physiologic and biochemical conditions of various diseases with the SARS-COV-2. In the case of cancer patients, intentional postponing of chemotherapy and surgery must be undertaken to curb the risk of COVID-19, especially in vulnerable places. As stated earlier, isolation and protective measures should be followed especially by and for cancer patients and cancer survivors.

Acknowledgements: We would like to express our gratitude to Graphic Era (Deemed to be) University, Dehradun for providing us technical support and constant encouragement throughout the process of this manuscript drafting.

\section{Conflict of interest: Nothing to declare.}

\section{REFERENCES}

Akula, S. M., Abrams, S. L., Steelman, L. S., Candido, S., Libra, M., Lerpiriyapo ng, K., ... McCubrey, J. A. (2020). Cancer therapy and treatments during COVID -19 era. Advances in Biological Regulation, 77, 100739. https://doi.org/10.1016/j .jbior.2020.100739

Bajgain, K. T., Badal, S., Bajgain, B. B., \& Santana, M. J. (2020). Prevalence of comorbidities among individuals with COVID-19: A rapid review of current liter ature. American Journal of Infection Control. S0196-6553(20):30637-30634. http s://doi.org/10.1016/j.ajic.2020.06.213

Burrell, L. M. (2005). Myocardial infarction increases ACE2 expression in rat an d humans: reply. European Heart Journal, 26(11), 1142-1143. https://doi.org/10. 1093/eurheartj/ehi275

Chen, L., Li, X., Chen, M., Feng, Y., \& Xiong, C. (2020). The ACE2 expression i $\mathrm{n}$ human heart indicates new potential mechanism of heart injury among patients infected with SARS-CoV-2. Cardiovascular Research, 116(6), 1097-1100. https ://doi.org/10.1093/cvr/cvaa078

Cheng, Y., Luo, R., Wang, K., Zhang, M., Wang, Z., Dong, L., ... Xu, G. (2020). Kidney disease is associated with in-hospital death of patients with COVID-19. K idney International, 97(5), 829-838. https://doi.org/10.1101/2020.02.18.2002324

Clark, A., Jit, M., Warren-Gash, C., Guthrie, B., Wang, H. H. X., Mercer, S. W., ... Ong, K. L. (2020). Global, regional, and national estimates of the population a 
$\mathrm{t}$ increased risk of severe COVID-19 due to underlying health conditions in 2020: a modelling study. The Lancet Global Health, 8(8), e1003-e1017. https://doi.org/ 10.1016/s2214-109x(20)30264-3

Dariya, B., \& Nagaraju, G. P. (2020). Understanding novel COVID-19: It impact on organ failure and risk assessment for diabetic and cancer patients. Cytokine \& Growth Factor Reviews, 53, 4352.https://doi.org/10.1016/j.cytogfr.2020.05.001

Guo, G., Ye, L., Pan, K., Chen, Y., Xing, D., Yan, K., ... Xue, X. (2020). New In sights of Emerging SARS-CoV-2: Epidemiology, Etiology, Clinical Features, Cli nical Treatment, and Prevention. Frontiers in Cell and Developmental Biology, 8 , 410. https://doi.org/10.3389/fcell.2020.00410

Harmer, D., Gilbert, M., Borman, R., \& Clark, K. L. (2002). Quantitative mRNA expression profiling of ACE 2, a novel homologue of angiotensin converting enz yme. FEBS Letters, 532(1-2), 107-110. https://doi.org/10.1016/s0014-5793(02)0 3640-2

Lambertini, M., Toss, A., Passaro, A., Criscitiello, C., Cremolini, C., Cardone, C. ,... Maio, M. D. (2020). Cancer care during the spread of coronavirus disease 20 19 (COVID-19) in Italy: young oncologists' perspective. ESMO Open, 5(2), e000 759. https://doi.org/10.1136/esmoopen-2020-000759

Larson, C., Oronsky, B., Goyal, S., Ray, C., Hedjran, F., Hammond, T. C., ... Re d, T. (2020). COVID-19 and cancer: A guide with suggested COVID-19 rule-out criteria to support clinical decision-making. Biochimica et Biophysica Acta (BBA) - Reviews on Cancer, 1874(2), 188412. https://doi.org/10.1016/j.bbcan.2020.188 412

Lee, L., Starkey, T., Cazier, J.-B., Kerr, R., \& Middleton, G. (2020). 1677MO C OVID-19 mortality in hospitalized cancer patients is not significantly affected by chemotherapy or other anti-cancer treatments. Annals of Oncology, 31, S994-S99 5. https://doi.org/10.1016/j.annonc.2020.08.1742

Li, Z., Wu, M., Yao, J., Guo, J., Liao, X., Song, S., ... Yan, J. (2020). Caution on Kidney Dysfunctions of COVID-19 Patients. SSRN Electronic Journa,1-25. https ://doi.org/10.2139/ssrn.3559601

Machhi, J., Herskovitz, J., Senan, A. M., Dutta, D., Nath, B., Oleynikov, M. D. ... Kevadiya, B. D. (2020). The Natural History, Pathobiology, and Clinical Mani festations of SARS-CoV-2 Infections. Journal of Neuroimmune Pharmacology, 1 5(3), 359-386. https://doi.org/10.1007/s11481-020-09944-5

Ruan, Q., Yang, K., Wang, W., Jiang, L., \& Song, J. (2020). Correction to: Clinic al predictors of mortality due to COVID-19 based on an analysis of data of $150 \mathrm{p}$ atients from Wuhan. China. Intensive Care Medicine, 46(6), 1294-1297. https://d oi.org/10.1007/s00134-020-06028-Z

Soy, M., Keser, G., Atagündüz, P., Tabak, F., Atagündüz, I., \& Kayhan, S. (2020 ). Cytokine storm in COVID-19: pathogenesis and overview of anti-inflammatory agents used in treatment. Clinical Rheumatology, 39(7), 2085-2094. https://doi.o $\mathrm{rg} / 10.1007 / \mathrm{s} 10067-020-05190-5$

TheActuary. The magazine of the Institute and Faculty of Actuaries. (2020 May). https://www.theactuary.com/

Wösten-van Asperen, R. M., Bos, A. P., Bem, R. A., Dierdorp, B. S., Dekker, T. van Goor, H., ... Lutter, R. (2013). Imbalance Between Pulmonary AngiotensinConverting Enzyme and Angiotensin-Converting Enzyme 2 Activity in Acute Re spiratory Distress Syndrome. Pediatric Critical Care Medicine, 14(9), e438-e441 https://doi.org/10.1097/pcc.0b013e3182a55735

Wösten-van Asperen, R. M., Lutter, R., Specht, P. A., Moll, G. N., van Woensel J. B., van der Loos, C. M., ... Bos, A. P. (2011). Acute respiratory distress syndro me leads to reduced ratio of ACE/ACE2 activities and is prevented by angiotens $\mathrm{n}-(1-7)$ or an angiotensin II receptor antagonist. The Journal of Pathology, 225(4) ,618-627.

World Health Organization (WHO). The Global Health Observatory. Noncommunicable diseases: Mortality.

https://www.who.int/data/gho/data/themes/topics/topic-details/GHO/ncdmortality

Wu, J., Deng, W., Li, S., \& Yang, X. (2020). Advances in research on ACE2 as a receptor for 2019-nCoV. Cellular and Molecular Life Sciences, 11:1-14. https://d oi.org/10.1007/s00018-020-03611-x

Wu, Z., \& McGoogan, J. M. (2020). Characteristics of and Important Lessons Fr om the Coronavirus Disease 2019 (COVID-19) Outbreak in China. JAMA, 323(1 3), 1239. https://doi.org/10.1001/jama.2020.2648

Xu, J., Fan, J., Wu, F., Huang, Q., Guo, M., Lv, Z., ... Jin, Y. (2017). The ACE2/ Angiotensin-(1-7)/Mas Receptor Axis: Pleiotropic Roles in Cancer. Frontiers in Physiology, 8:1-8. https://doi.org/10.3389/fphys.2017.00276

Xu, X., Chen, P., Wang, J., Feng, J., Zhou, H., Li, X., ... Hao, P. (2020). Evoluti on of the novel coronavirus from the ongoing Wuhan outbreak and modeling of it s spike protein for risk of human transmission. Science China Life Sciences, 63(3) , 457-460. https://doi.org/10.1007/s11427-020-1637-5

Yan, C. H., Faraji, F., Prajapati, D. P., Boone, C. E., \& DeConde, A. S. (2020). A ssociation of chemosensory dysfunction and COVID-19 in patients presenting wi th influenza-like symptoms. International Forum of Allergy \& Rhinology, 10(7), 806-813. https://doi.org/10.1002/alr.22579

Yang, X., Yu, Y., Xu, J., Shu, H., Xia, J., Liu, H., ... Shang, Y. (2020). Clinical c ourse and outcomes of critically ill patients with SARS-CoV-2 pneumonia in Wu han, China: a single-centered, retrospective, observational study. The Lancet Res piratory Medicine, 8(5), 475-481. https://doi.org/10.1016/s2213-2600(20)300795

Yu, J., Ouyang, W., Chua, M. L. K., \& Xie, C. (2020). SARS-CoV-2 transmissio $\mathrm{n}$ in cancer patients of a tertiary hospital in Wuhan. JAMA Oncolology, 6(7):1108 -1110.https://doi.org/10.1101/2020.02.22.20025320

Zaki, N., Alashwal, H., \& Ibrahim, S. (2020). Association of hypertension, diabet es, stroke, cancer, kidney disease, and high-cholesterol with COVID-19 disease s everity and fatality: a systematic review. Diabetes and Metabolic Syndrome: Clin ical Research and Reviews, 14(5):1133-1142.https://doi.org/10.1101/2020.06.16. 20132639

Zhang, H., Penninger, J. M., Li, Y., Zhong, N., \& Slutsky, A. S. (2020). Angioten sin-converting enzyme 2 (ACE2) as a SARS-CoV-2 receptor: molecular mechani sms and potential therapeutic target. Intensive Care Medicine, 46(4), 586-590. ht tps://doi.org/10.1007/s00134-020-05985-9

Zhang, J., Dong, X., Cao, Y., Yuan, Y., Yang, Y., Yan, Y., ... Gao, Y. (2020). Cl inical characteristics of 140 patients infected with SARS-CoV-2 in Wuhan, China . Allergy, 75(7), 1730-1741. https://doi.org/10.1111/all.14238 